\title{
Effect of traditional sequential medial soft tissue release on knee kinematics and alignment
}

\begin{abstract}
Traditionally sequential medial soft tissue release is performed for balancing varus knees in total knee arthroplasty. Its effects on kinematics have been described in extension and $90^{\circ}$ flexion in coronal plane but not through complete range of flexion. This study describes its effects on knee kinematics, throughout the range of motion of the knee, studied using a computer navigation system. Twelve cadaveric knees were studied. Femoro-TibialMechanical-Angle (FTMA) was studied in extension, $0^{\circ}, 15^{\circ}, 30^{\circ}, 45^{\circ}, 60^{\circ}, 90^{\circ}$ and maximum flexion. Sequential medial release was performed in 7 steps, described by Luring et al. ${ }^{1}$ At each step FTMA was measured with and without varus and valgus stressing. A 10 Newton Meter (NM) moment was applied for varus and valgus stress.
\end{abstract}

Most of the initial release steps had little effect on FTMA without force application, especially in the initial $60^{\circ}$ of flexion. Application of varus force demonstrated very small changes. Even the application of valgus force demonstrated little change in initial arc of flexion until step 5 in the release was reached.

Our study concludes that the present sequence of medial release may not be correct and should be investigated further to modify the sequence for sequential medial release for balancing in varus knees.

Keywords: Tibio-femoral, Parapatellar, Coronal angulation, Angulation, Tensioner, Tibiofemoral, Corroborates, Flexion, Deformity, Kinematic, Phenomenon
Volume 4 Issue $6-2016$

\author{
Deep K,' Duffy S, ${ }^{2}$ Sharma M,' Goudie ST, I \\ Khan MS,' Payne A² \\ 'Department of Orthopaedics, Scotland \\ ${ }^{2}$ University of Glasgow, Scotland \\ Correspondence: Kamal Deep, Department of Orthopaedics, \\ Golden Jubilee National Hospital, Agamemnon Street, \\ Clydebank, West Dunbartonshire, G8I 4DY, Scotland, Tel 44 \\ (0) I4I 95। 5392, Fax 44 (0) I4I 95। 508।, \\ Emailmrdeep@gmail.com, kamal.deep@gjnh.scot.nhs.uk
}

Received: February 28, 2016 | Published: April 23, 2016
Abbreviations: FTMA, Femoro-Tibial-Mechanical-Angle; NM, Newton Meter; TKR, Total Knee Replacement; PCL, Posterior Cruciate Ligament

\section{Introduction}

Soft tissue balancing is an important aspect of total knee replacement (TKR) surgery. Numerous soft tissue balancing release sequences have been published in literature. ${ }^{1-6}$ Matseuda et al. ${ }^{5}$ had proposed a sequential release of medial structures as opposed to Insall et al. ${ }^{6}$ who proposed release of all medial structures as one. ${ }^{5,6}$ Traditionally sequential medial soft tissue release is performed for balancing in varus deformity to correct the alignment in TKR surgery. Its effects on kinematics and knee alignment as measured by femorotibial mechanical-angle (FTMA) have been described in extension and $90^{\circ}$ flexion in coronal plane. ${ }^{1}$ However most studies have missed what happens when the knee flexes from 0 to 90 degrees. ${ }^{1,4,5}$ Traditionally it would be impossible to note little changes produced by these releases during the TKR surgery using conventional instruments. With advent of computer assisted surgery it is now possible to objectively assess the effects of releases of minor structures. The technology has now shown that the alignment of the knee is not static and is dynamic in nature. ${ }^{7}$ It changes during the flexion of the knee. ${ }^{8}$ So a global release of structures sequentially or together will have various effects on alignment depending on the structure released and position of flexion at the time. Studies have suggested that most traditional releases are not required or should be tailored to the specific knees depending on their kinematics. ${ }^{9}$ This study was designed to analyse sequential medial soft tissue release effects on knee kinematics throughout flexion. As during TKR surgery it will not be appropriate to do sequential release of all the structures for purpose of study, it was designed to study the effects of these in cadaveric knees. The aim was to look at deviation of FTMA in coronal plane with traditional sequential medial release with and without measured stress applied in varus and valgus direction at each point of measurement through the range of flexion.

\section{Material and methods}

This research was completed at the Laboratory of Human Anatomy at the University of Glasgow, under the 2006 (Scotland) Amendment to the Human Anatomy Act and therefore did not require review by an Ethics Committee.

12 lower limbs from 7 fresh frozen cadavers were studied which were supplied by the department of anatomy. Mean age was $88 \mathrm{yrs}$ (range 84-93), four were females and three were males. The average time since death was 9 months (range 8 to 10 months). Nine of the knees had signs of arthritis affecting the tibio-femoral and patello femoral articular cartilage, in one knee only the tibio-femoral joint was arthritic (i.e. the femoral trochlea was spared) and the remaining two knees were normal. None of the knees had advanced degree of osteoarthritis or varus deformity. There was no restriction of motion in any of the knees. None of the cadaveric specimens had previous surgery of the hip, knee or ankle. Three legs had loosening of pins/ pin cut out due to osteoporotic bones and the study was abandoned in those cases leaving behind nine lower limbs which were included in the study.

The FTMA and stress kinematics were studied using a computer navigation system (Orthopilot, BBraun Aesculap, Tuttlingen, Germany). Rigid bodies with passive reflective ball markers were fixed to femur and tibia (Figure 1). The knee was exposed with medial parapatellar approach as in TKR surgery but with no disturbance to the collateral ligaments or medial structures. The anatomy of the knee was registered using an infra red waves based passive tracker navigation system. Hip knee and ankle joint centres were generated kinematically through movements of the joints. Knee and ankle centres were validated by registration of anatomical points in addition. The software then generated the FTMA by connecting the centres of hip, knee and ankle (Figure 2). The FTMA measurements in both coronal and sagittal planes were extracted from the recorded computer navigation files for analysis. Data in the computer files were recorded 
to the nearest $0.5^{\circ}$. FTMA was studied in extension, $0^{\circ}, 15^{\circ}, 30^{\circ}, 45^{\circ}$, $60^{\circ}, 90^{\circ}$ and maximum flexion. A hole was drilled in medio-lateral direction in the tibia at 25 centimetre distance from joint line. And hooks were attached to the tibia at this point medially and laterally for application of varus and valgus stress (Figure 1). A digital loading device was attached to the hook and used to apply a load of $4 \mathrm{Kg}$ to apply the moment 10 Newton Meters (NM) in varus and valgus directions.

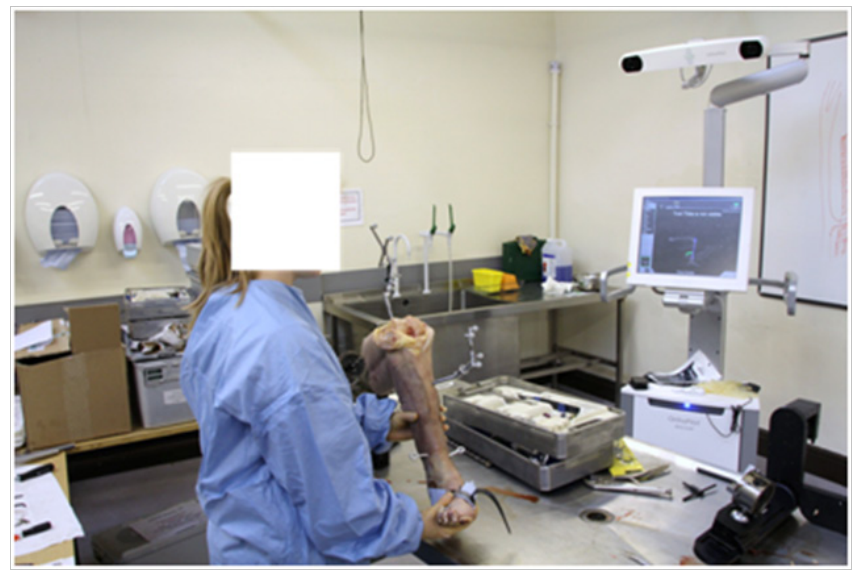

Figure I Set up demonstrating the rigid body markers attached to the tibia and femur and computer navigation system. Also noted is the hooks inserted in the tibia for varus and valgus stress application.

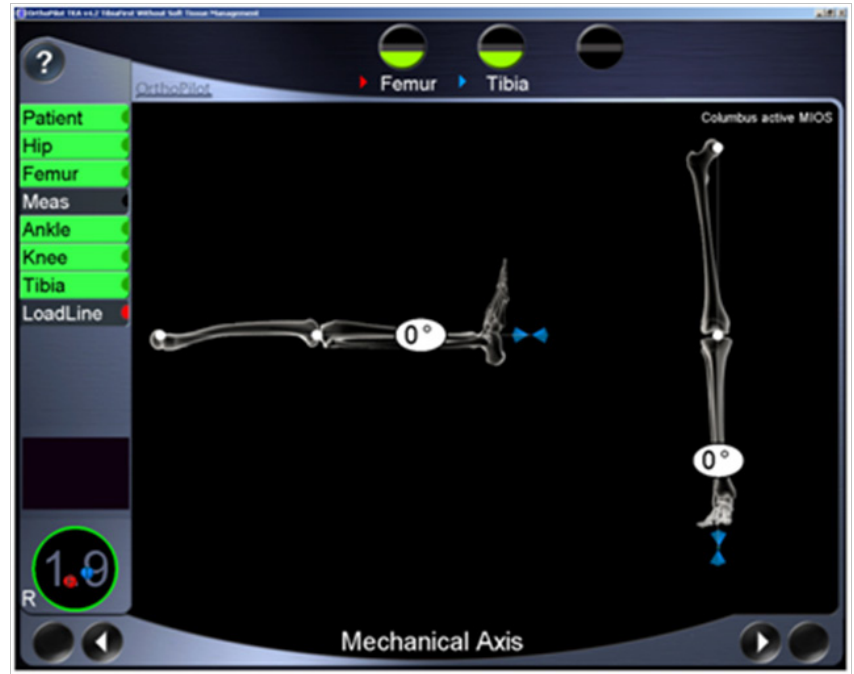

Figure 2 Computer screen showing the FTMA in coronal and sagittal plane.

Sequential medial release was performed in 7 steps as described by Luring et al. ${ }^{1}$ The sequential steps were Step 1: $2 \mathrm{~cm}$ release of antero medial tibial sleeve, Step 2: Postero-medial release, Step 3: $4 \mathrm{~cm}$ medial sleeve release, Step 4: $6 \mathrm{~cm}$ medial sleeve release, Step 5: Deep medial collateral ligament release, Step 6: posterior cruciate ligament (PCL) release of medial half only, Step 7: release of entire PCL.

At each step FTMA was measured with and without stressing at each point of flexion. A $10 \mathrm{NM}$ force was applied for varus and valgus stress at each point in extension, $0^{\circ}, 15^{\circ}, 30^{\circ}, 45^{\circ}, 60^{\circ}, 90^{\circ}$ and maximum flexion and FTMA was recorded.

\section{Results}

Most of the initial release steps had very little effect on FTMA without force application, especially in the initial $60^{\circ}$ of flexion. This is clearly evident on the Figures 3.1 to 3.7 , which show the various steps of releases with no stress applied.

Application of varus force demonstrated very small changes at all points of flexion. This is clearly evident on the Figures 4.1 to 4.7, which show the various steps of releases with $10 \mathrm{NM}$ varus stress applied.

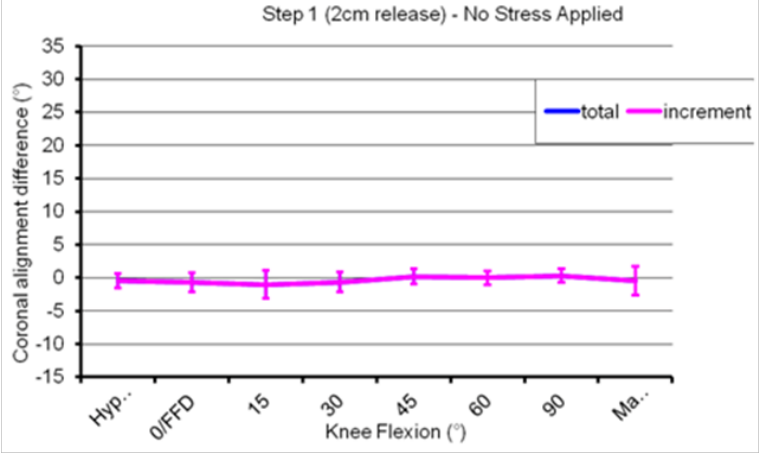

Figure 3 Show the various steps of releases with no stress applied.

Figure 3.I Step I ( $2 \mathrm{~cm}$ release)- No Stress Applied.

$X$ Axis denotes the flexion position from hyperextension position through $0^{\circ}$, $15^{\circ}, 30^{\circ}, 45^{\circ}, 60^{\circ}, 90^{\circ}$ and maximum flexion positions.

$\mathrm{Y}$ Axis denotes the deviation in degrees in FTMA with blue lines depicting total change in FTMA from start position and purple denoting increment over the last step of release.

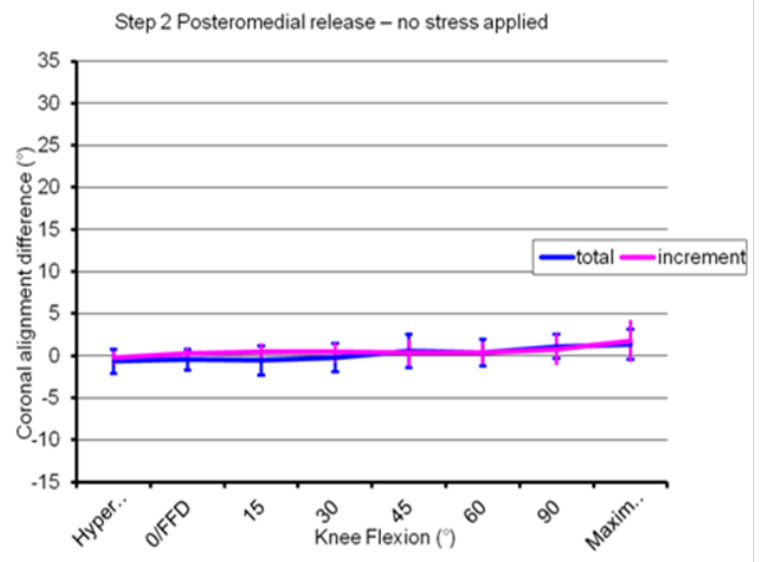

Figure 3.2 Step 2 Posteromedial release-no stress applied.

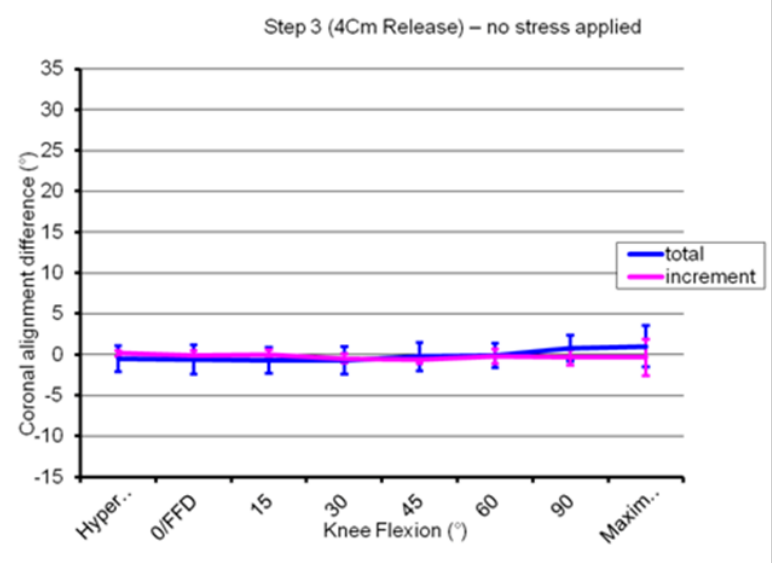

Figure 3.3 Step 3 ( $4 \mathrm{~cm}$ release)-no stress applied. 


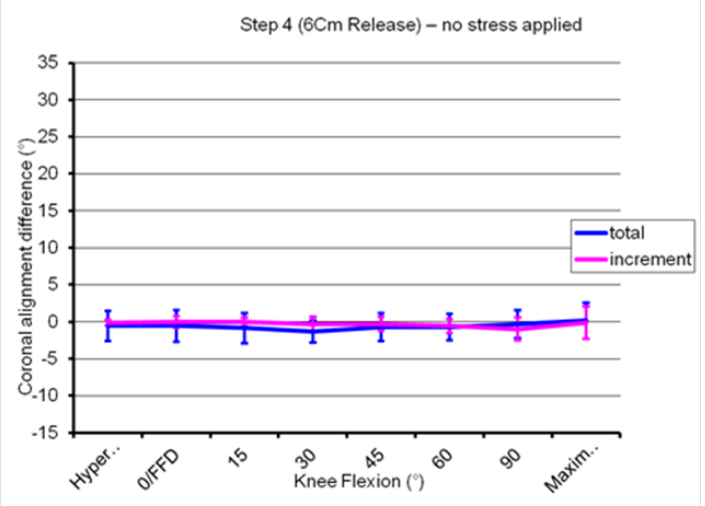

Figure 3.4 Step 4 (6cm Release)-no stress applied.

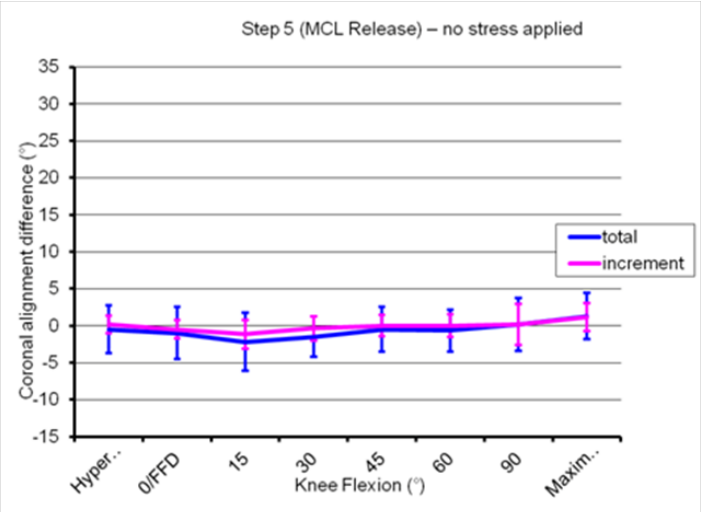

Figure 3.5 Step 5 (MCL Release)-no stress applied.

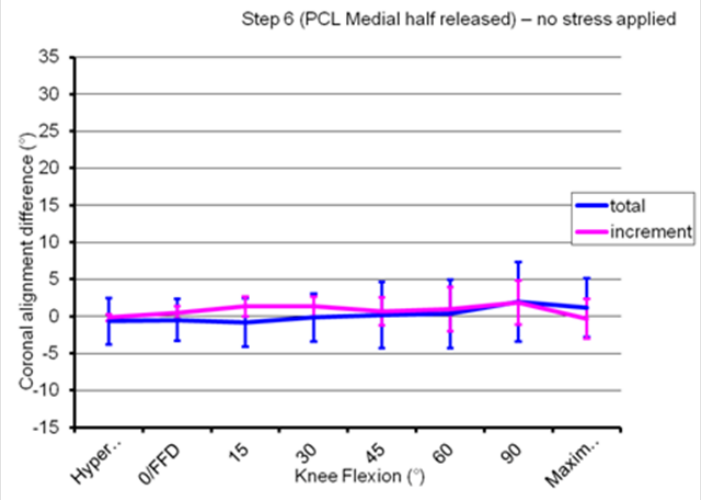

Figure 3.6 Step 6 (PCL Medial half released)-no stress applied.

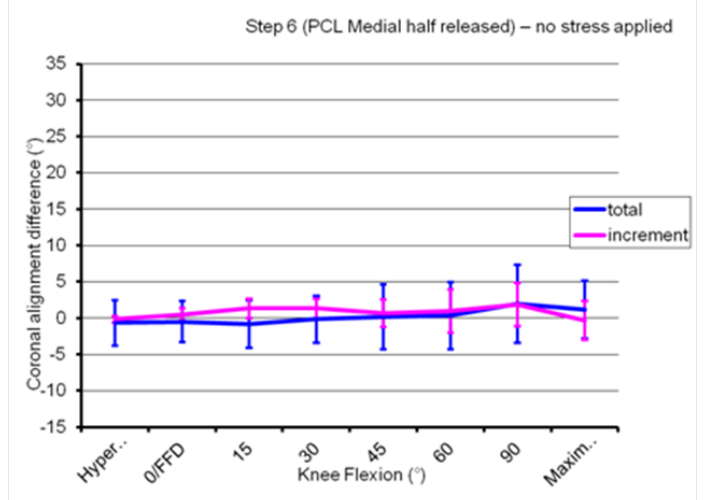

Figure 3.7 Step 7 (PCL Released)-no stress applied.

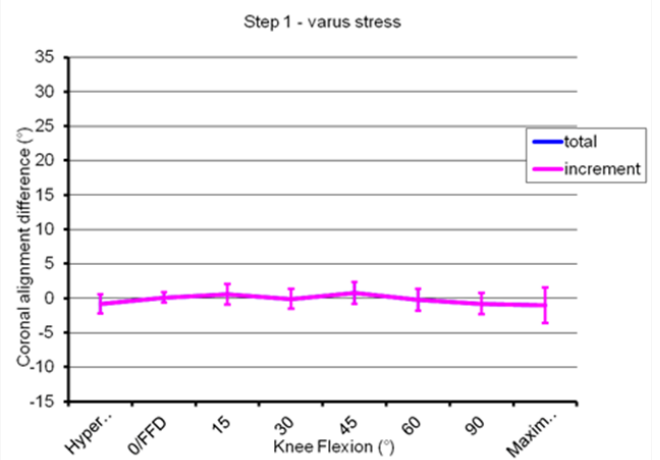

Figure 4 IONM varus stress applied.

Figure 4.I Step I-Varus stress.

$\mathrm{X}$ Axis denotes the flexion position from hyperextension position through $0^{\circ}$, $15^{\circ}, 30^{\circ}, 45^{\circ}, 60^{\circ}, 90^{\circ}$ and maximum flexion positions.

$Y$ Axis denotes the deviation in degrees in FTMA with blue lines depicting total change in FTMA from start position and purple denoting increment over the last step of release.

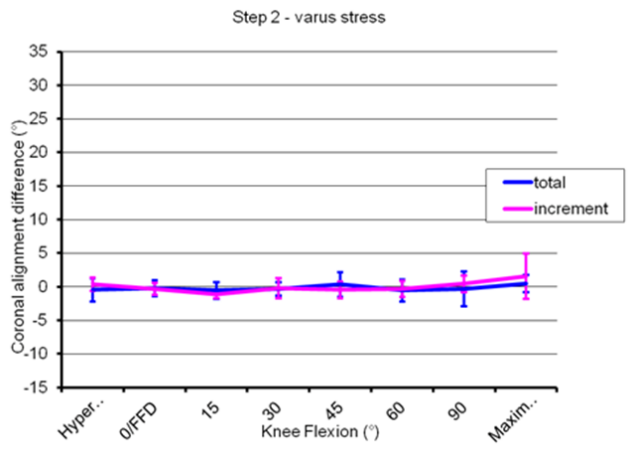

Figure 4.2 Step 2-Varus stress.

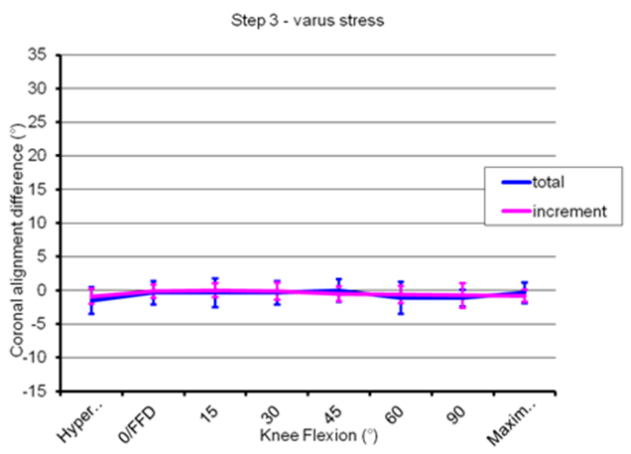

Figure 4.3 Step 3-Varus stress.

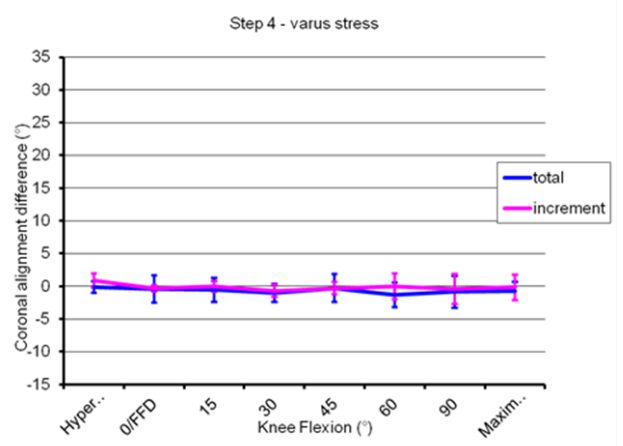

Figure 4.4 Step 4-Varus stress.

Citation: Deep K, Duffy S, Sharma M, et al. Effect of traditional sequential medial soft tissue release on knee kinematics and alignment. MOJ Orthop Rheumatol. 2016;4(6): 140-I45. DOI: 10.15406/mojor.2016.04.00156 


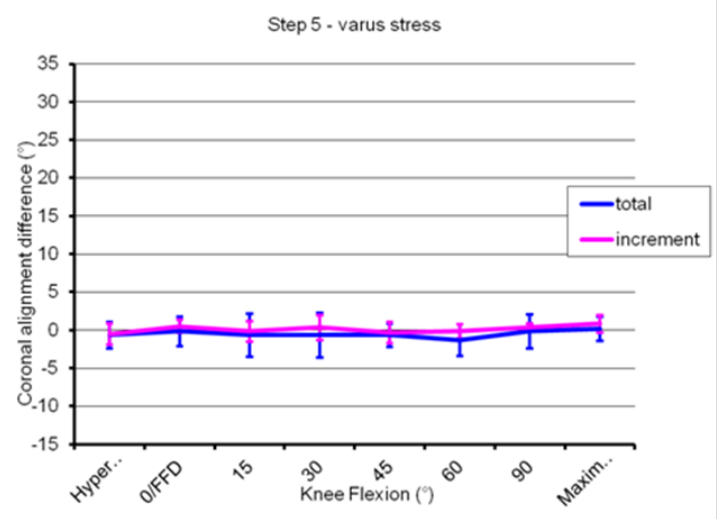

Figure 4.5 Step 5-Varus stress.

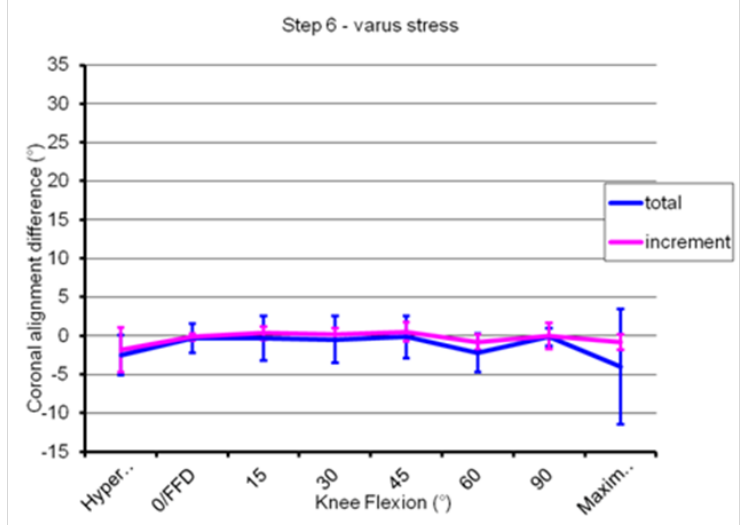

Figure 4.6 Step 6-Varus stress.

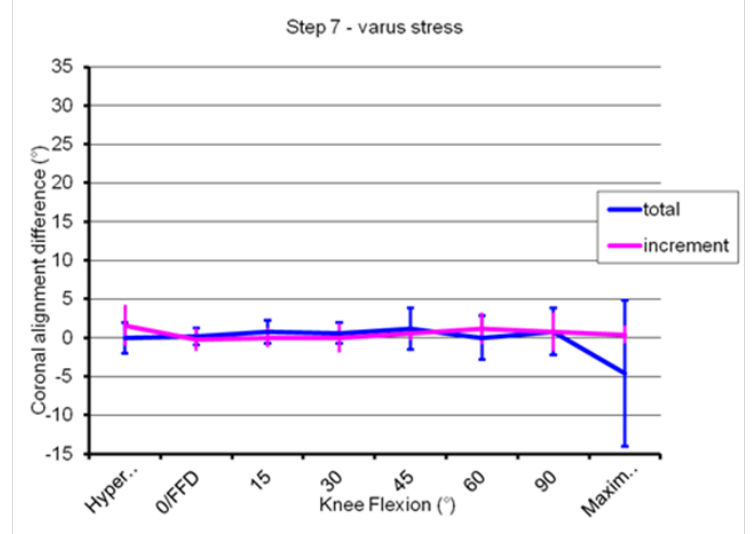

Figure 4.7 Step 7-Varus stress.

Application of valgus force demonstrated little change in initial arc of flexion until step 5 was reached. After the release of the deep MCL, a significant change in coronal plane alignment on giving a valgus stress was seen, at 60 degree of knee flexion. The sequential change in FTMA building on the last step position was also maximum after step 5 release at 60 degrees of flexion. This is clearly evident on the Figures 5.1 to 5.7, which show the various steps of releases with $10 \mathrm{NM}$ valgus stress applied.

Table 1 shows total change in FTMA from initial position (mean) with $10 \mathrm{NM}$ valgus force application at each step of release through range of flexion
Table I Mean total change in FTMA in degrees, with sequential medial release steps with IONM valgus force applied at each point of flexion

\begin{tabular}{llllllll}
\hline Limb Position & StepI & Step2 & Step3 & Step4 & Step5 & Step6 & Step7 \\
\hline Hyperextension & -0.1 & 0.2 & 0.1 & 0.4 & 0.7 & 1.7 & 4.4 \\
$0^{\circ}$ Flexion & -0.1 & 0.6 & 0.6 & 0.6 & 2.5 & 3.5 & 6.6 \\
$15^{\circ}$ Flexion & -0.3 & 0.4 & 0.6 & 1.4 & 3.8 & 5.3 & 8.8 \\
$30^{\circ}$ Flexion & 0.6 & 1.2 & 1.5 & 2 & 5.8 & 8.5 & 11 \\
$45^{\circ}$ Flexion & 0.8 & 1.3 & 1.6 & 2.3 & 6 & 10.5 & 12.2 \\
$60^{\circ}$ Flexion & 0.9 & 1.3 & 2.2 & 3 & 5.5 & 11 & 15.2 \\
$90^{\circ}$ Flexion & 0.1 & 1.6 & 1.6 & 2.3 & 4.5 & 12.2 & 22.4 \\
Maximum Flexion & 1.3 & 3.1 & 4.5 & 1.7 & 5.1 & 15 & 24.4 \\
\hline
\end{tabular}

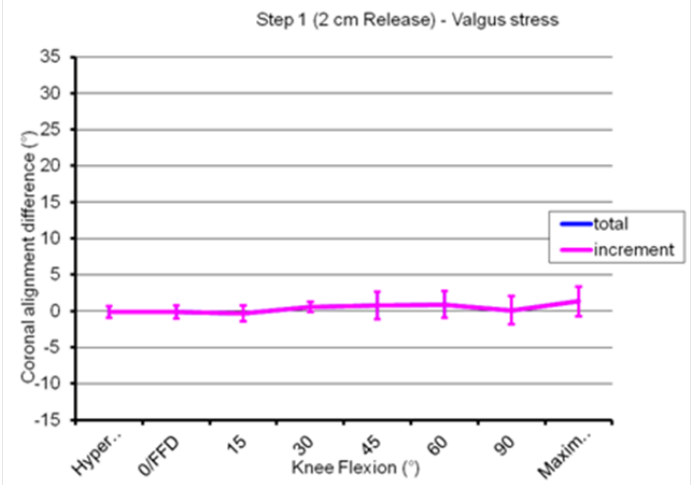

Figure 5 IONM valgus stress applied.

Figure 5.I Step I (2cm Release)-Valgus stress.

$X$ Axis denotes the flexion position from hyperextension position through $0^{\circ}$, $15^{\circ}, 30^{\circ}, 45^{\circ}, 60^{\circ}, 90^{\circ}$ and maximum flexion positions.

$Y$ Axis denotes the deviation in degrees in FTMA with blue lines depicting total change in FTMA from start position and purple denoting increment over the last step of release.

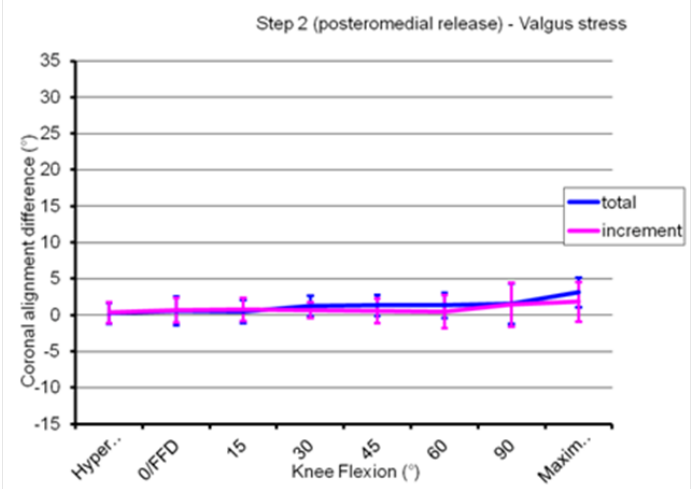

Figure 5.2 Step 2 (Posteromedial release)-Valgus stress.

Step $3(4 \mathrm{~cm}$ release) - valgus stress

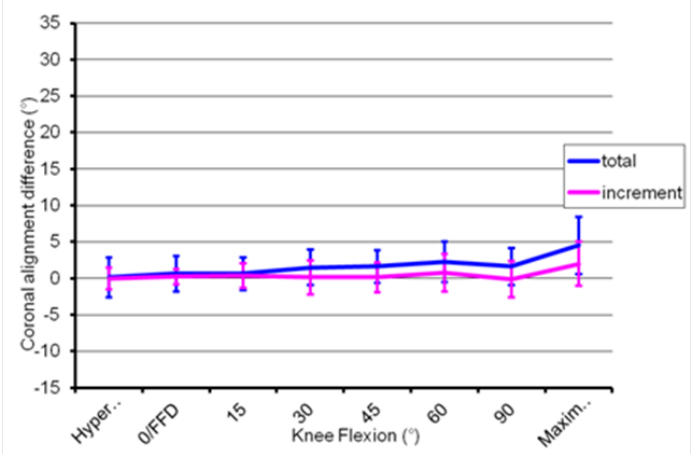

Figure 5.3 Step $3(4 \mathrm{~cm}$ release)-Valgus stress. 


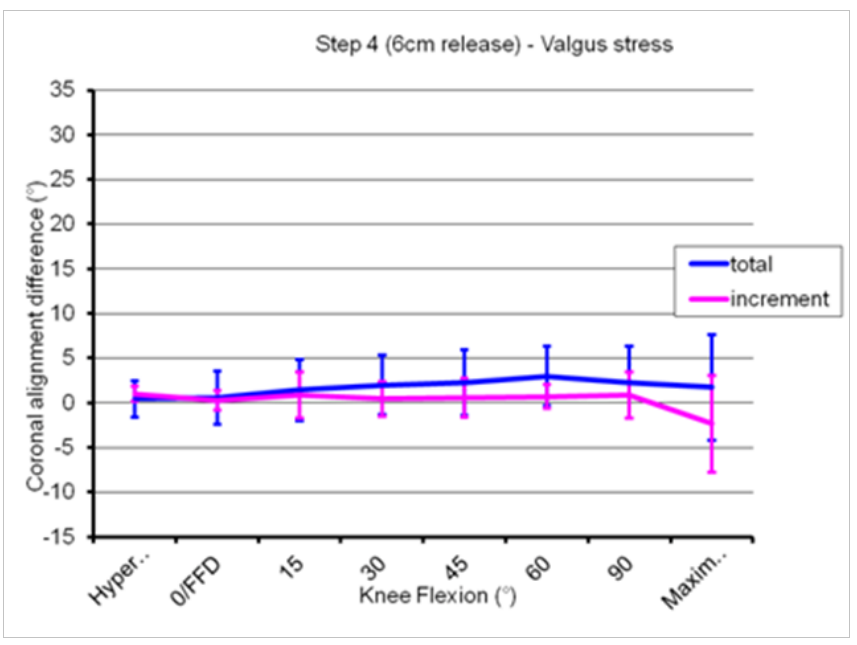

Figure 5.4 Step 4 (6cm release)-Valgus stress.

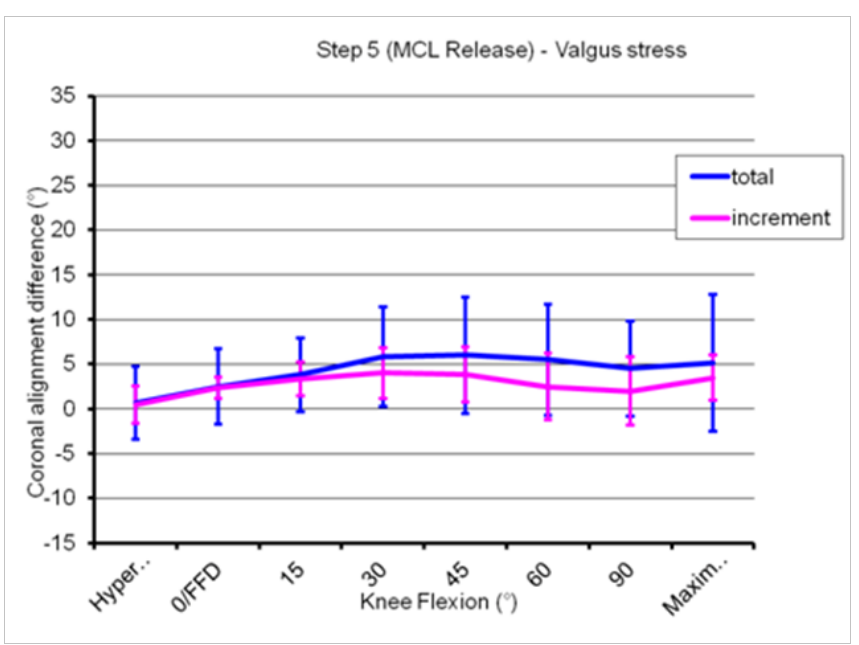

Figure 5.5 Step 5(MCL Release)-Valgus stress.

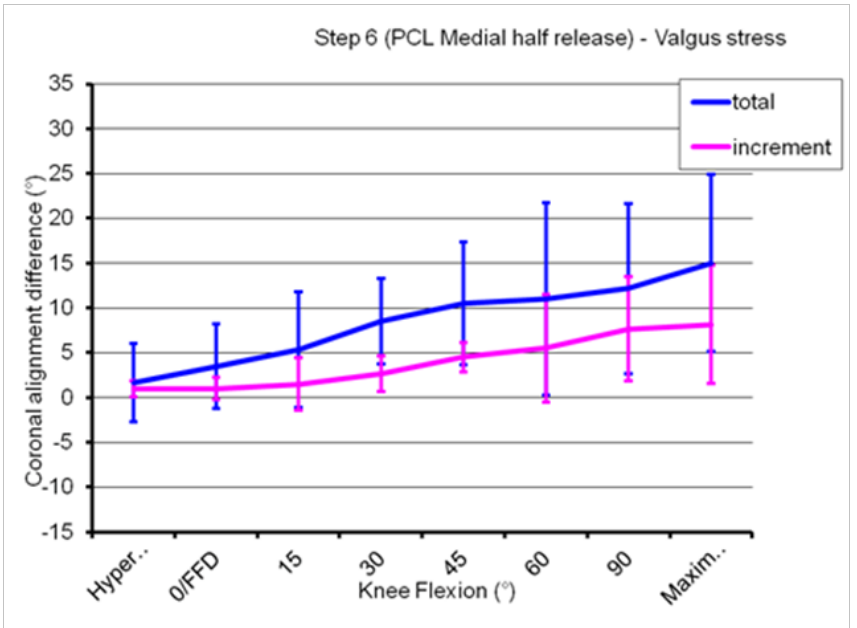

Figure 5.6 Step 6 (PCL Medial half release)-Valgus stress.

\section{Discussion}

Our study shows that the initial steps of the release make little difference to the knee alignment in initial range of flexion even with applied valgus stress force. Thus the present sequence of medial release may not be correct and should be further investigated to modify the sequence for soft tissue balancing in TKR surgery for varus deformity.

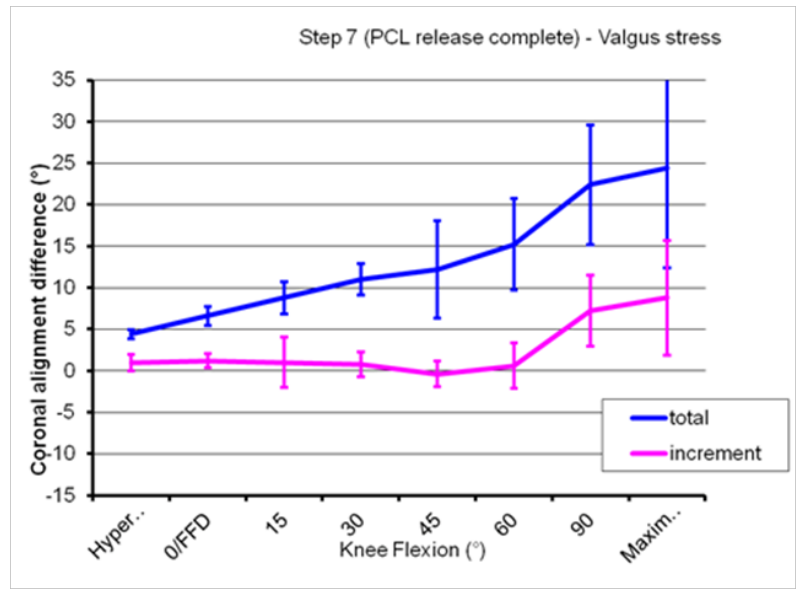

Figure 5.7 Step 7 (PCL release complete)-Valgus stress.

Our study has certain limitations. The number of cadavers included in the study is small. Though the cadavers were with supple tissues which felt normal, the tension of soft tissues in cadavers may differ from those in living and the same results of release may not apply in the living human beings. Our study points to the fact that the present sequence of medial soft tissue releases may not be correct, but it does not give what the correct sequence of medial soft tissues release should be. Our cadavers did not have severe arthritic knees where the tissue balancing may be needed. Also we released the soft tissues in intact knees rather than replaced knees, so the observations made in this study should be considered with caution as the two knees may not behave the same way.

Traditional sequential medial release for balancing in total knee replacement is based mainly on subjective experience of surgeons. For soft tissue balancing until now surgeons have mainly considered balancing at 0 and 90 degrees flexion with stress forces applied at these points especially when using the gap resection techniques..$^{1,4,5}$ The middle part of the motion has been mostly neglected due to unavailability of any practical measurement devices. It is now known that the coronal plane alignment can change throughout the arc of flexion. ${ }^{8,10}$ Moreover coronal plane alignment seen or measured intraoperatively during TKR is not necessarily the same as the deformity seen on standing long-leg radiograph either pre-or post-operatively. ${ }^{11}$ Phenomenon of mid flexion instability is not uncommon. ${ }^{12}$ Computer navigation has made it possible to monitor the kinematics throughout the range of flexion..$^{13}$ The traditional sequential release and balancing methods were designed when there was not much insight into middle range of the flexion movement. Now with objective measurement devices being available, we need to revisit the techniques.

Numerous cadaveric studies comparing ligament balancing techniques and medial soft tissue release sequence have been conducted in literature. Mihalko et al. ${ }^{2}$ conducted a cadaveric study and compared two different ligament balancing technique in knees for obtaining a balanced flexion and extension gap. ${ }^{2}$ They obtained the same findings for the release of PCL, a similar result to Luring et al. ${ }^{1}$ One limitation of that study was their use of cadaveric knees without hips and ankle joints. So the assessment of coronal plane alignment was not possible. ${ }^{2}$ Matseuda et al. ${ }^{5}$ studied the relationship between the individual steps in medial or lateral soft tissue release sequence with changes in medial and lateral tibiofemoral gaps and changes in 
coronal angulation caused by $10 \mathrm{Nm}$ varus or valgus moments force in extension and 90 degree flexion. ${ }^{5}$ They found a significant increase in coronal angulation after release of $8 \mathrm{~cm}$ of anteromedial sleeve. A limitation of that study, like many others, was that they conducted their measurements in extension and 90 degree flexion but middle part of flexion remained ignored. ${ }^{5}$ Their study differs significantly from ours as we found no significant change in coronal angulation (FTMA) after release of antero-medial sleeve up to $6 \mathrm{~cm}$.

Luring et al. ${ }^{1}$ also studied the effect of medial soft tissue release on normal knees. They used a tensioner to measure flexion and extension gaps with the surgeons producing a manual tension which is subjective and may be inconsistent. We have used a consistent 10NM force to apply stress in our study. Luring et al. ${ }^{1}$ obtained maximum increase in medial tibiofemoral gap and leg axis at step 4 and further in their study, whereas we found no major change in alignment till about 60 degrees of flexion with valgus force applied till step 5 (release of deep MCL) of the release was reached. Both Luring's and present studies demonstrate that first three steps of medial release have little if any effect on FTMA. Luring et al. ${ }^{1}$ started to see effect of their release in step 4 while we found this to happen after step 5 .

Schwarzkopf et al. ${ }^{11}$ used navigation for dynamic evaluation of knee alignment throughout the full range of motion. ${ }^{10}$ They studied 65 TKR using navigation and classified stress-strain curve generated throughout range of motion-13\% diagonal, $18.5 \%$ C-shaped, $43.5 \%$ comma shaped, and $25 \%$ S-shaped. Patterns were reproduced with cadaveric knees by recreating specific contracture constellations. A tight posterior capsule gave an S-shaped curve, a tight lateral collateral ligament gave a $\mathrm{C}$-shaped curve, tight medial collateral ligament gave a diagonal curve, and a tight posterior lateral corner gave a commashaped curve. Release of the specific contractures in cadaver knees resulted in correction of all patterns of deformity as measured by CAS. They introduced a classification system to match individual and combined soft tissue pathological contractures to specific kinematic curves obtained through routine knee CAS preparation..$^{10}$ Their study may shed light on future soft tissue releases but does not define the release sequence which should be followed. Deep et al. ${ }^{7}$ has given navigation based analysis and classification of knee kinematics. ${ }^{8}$ In their study on 283 TKR they found that the majority of knees did not behave as true varus or valgus knees. They classified the deformity into four groups depending upon the behaviour of the knee in coronal plane as it moves from extension to flexion. ${ }^{8}$ They concluded that traditional release of medial or lateral soft tissue structures without realising the true picture of what happens when the knee is flexed may not be correct. Some of the releases performed conventionally may not be required or need to be modified depending upon the knee kinematics. ${ }^{8-10}$

The traditional sequential release and balancing methods were designed when there was not much insight into middle range of the flexion movement. Now with objective measurement devices being available we need to revisit the techniques. Present study shows that the initial steps of the traditional sequential release make little difference to the knee alignment in initial range of flexion even with applied valgus stress force and further corroborates with the findings of previous studies. ${ }^{1,9,10}$ Thus the present sequence of medial release may not be correct and should be further investigated to modify the sequence for soft tissue balancing in TKR surgery for varus knees.

\section{Acknowledgements}

Authors would like to thank Dr Angela Deakin, $\mathrm{PhD}$ for her help with statistics and graphics.

\section{Conflicts of interest}

None.

\section{References}

1. Luring C, Huffner T, Perlick, et al. The effectiveness of sequential medial soft tissue release on coronal alignment in Total Knee Arthroplasty. $J$ Arthroplasty. 2006;21(3):428-434

2. Mihalko WM, Whiteside LA, Krackow KA. Comparison of ligamentbalancing techniques during total knee arthroplasty. J Bone Joint Surg Am. 2003;85(Suppl 4):132-135

3. Faris PM. Soft tissue balancing and total knee arthroplasty. In: Fu FH, et al. (eds.), Knee surgery. Williams and Wilkins, Baltimore, USA. 1994.

4. Krakow KA, Mihalko WM. The effect of medial release on flexion and extension. Gaps in cadaveric knees: implications for soft tissue balancing in total knee arthroplasty. Am J Knee Surg. 1999;12(4):222-228.

5. Matsueda M, Gengerke TR, Murphy M, et al. Soft tissue release in total knee arthroplasty. Cadaver study using knees without deformities. Clin Orthop Relat Res. 1999;366:264-273.

6. Insall JN, Scott WN, Ranawat CS. The total condylar knee prosthesis: a report of two hundred and twenty cases. J Bone Joint Surgery Am. 1979;61(2):173-180.

7. Deep K, Eachempati KK, Apsingi S. The dynamic nature of knee alignment and variations in normal knees. Bone Joint $\mathrm{J}$. 2015;97(4):498-502.

8. Deep K, Picard F, Baines J, et al. An analysis of knee kinematics with computer navigation: A classification of kinematics and nomenclature for arthritis of knee. J Bone Joint Surg Br. 2011;93B(SUPP III):391.

9. Goudie S, Deep K. Collateral soft tissue release in primary total knee replacement. Comput Aided Surg. 2014;19(1-3):29-33.

10. Schwarzkopf R, Hadley S, Abbasi M, et al. Computer-assisted surgery patterns of ligamentous deformity of the knee: a clinical and cadaveric study. J Knee Surg. 2013;26(4):233-238.

11. Willcox NMJ, Clarke JV, Smith BRK, et al. A comparison of radiological and computer navigation measurements of lower limb coronal alignment before and after total knee replacement. J Bone Joint Surg Br. 2012;94(9):1234-1240.

12. Yercan HS, Selmi AS, Sugun TS, et al. Tibiofemoral instability in total knee replacement: a review, Part 1: Basic principles and classification. Knee. 2005;12(4):257-266.

13. Deep K, Picard F, Clarke JV. Dynamic knee alignment and collateral knee laxity and its variations in normal humans. Front Surg. 2015;2:62. 